\section{LA LUCHA POR LA VIVIENDA EN ESPAÑA (2009-2019): DESDE LA CALLE A LAS INSTITUCIONES}

Eva Álvarez de Andrés ${ }^{1}$ y Harry Smith ${ }^{2}$

\section{Resumen}

La crisis financiera entre 2007 y 2008, y las políticas nacionales de mercantilización y de financiarización de la vivienda, han dejado a millones de familias sin hogar en todo el mundo. Este trabajo analiza el proceso que se ha llevado a cabo en España en la última década (2009-2019) de lucha por hacer efectivo el derecho la vivienda, desde la toma de la calle a la toma de las instituciones, empleando el enfoque de la teoría de la estructuración de Giddens para valorar la capacidad de estos procesos para transformar las relaciones de autoridad, la asignación de recursos y los sistemas de significado. El análisis de la PAH y Podemos

\section{THE STRUGGLE FOR HOUSING IN SPAIN (2009-2019): FROM THE STREET TO THE INSTITUTIONS}

\author{
Eva Álvarez de Andrés y Harry Smith
}

\section{Abstract}

The financial crisis of 2007 and 2008, and the national policies of commodification and financialization of housing, have made millions of households homeless around the world. This paper analyses the process that have taken place in Spain over the last decade (2009-2019) in a 'struggle' to enact the right to housing - a process that has gone from 'taking the street' to 'taking the institutions'. This is analyzed through the lens of Giddens's theory of structuration, with the aim of assessing the capacity of these processes to transform relationships with authority, the allocation of resources, and systems of meaning. Analysis of PAH and Podemos shows how 
muestra cómo consiguieron cambiar el imaginario colectivo y proponer alternativas a nivel político, pero también la limitación en conseguir una transformación de la política de vivienda. Muestra además que la gente común puede convertirse en sujeto político y desafiar el discurso capitalista de que los derechos de la propiedad deben anteponerse al bien común y más concretamente al derecho a la vivienda. Los resultados pueden ser de utilidad en contextos similares de crisis económica, deslegitimación política y vulneración del derecho a la vivienda, tales como Grecia e Irlanda.

\section{PALABRASCLAVE: MOVILIZACIÓN SOCIAL;DERECHO} A LA VIVIENDA; INSTITUCIONES ESPAÑOLAS.

Recibido: 29-06-2019

Aceptado: 16-10-2019 they have changed the collective imagination and proposed alternatives at a political level, but also the limits in achieving a transformation of housing policy. The case of Spain shows that 'common people' can acquire political agency and challenge the capitalist discourse that property rights should be prioritized over the common good, and specifically over the right to housing. This analysis offers lessons that could help understand similar contexts of economic crisis, political delegitimization and breach of the right to housing, such as Greece and Ireland.

\section{KEYWORDS: SOCIAL MOBILIZATION; RIGHT TO} HOUSING; SPANISH INSTITUTIONS.

Received: 29-06-2019

Accepted: 16-10-2019
España. Profesora asistente Escuela Técnica Superior de Arquitectura, Universidad Politécnica de Madrid, http://orcid. org/0000-0002-7370-7053. Correo electrónico: eva.alvarez@ upm.es
2 Reino Unido. Profesor asociado Centre for Environment \& Human Settlements; The Urban Institute, School of Energy, Geoscience, Infrastructure and Society, Heriot-Watt University, http:// orcid.org/0000-0003-0922-5575. Correo electrónico:h.c.smith@ hw.ac.uk 


\section{Introducción}

La crisis financiera y las políticas urbanas en su conjunto han dejado a millones de personas sin hogar en todo el mundo. Rolnik (2018) sostiene que, globalmente, las ciudades se han vendido al sector inmobiliario-financiero, un proceso favorecido por un conjunto de políticas nacionales que han hecho de la vivienda un elemento clave de extracción de renta, ganancia financiera y acumulación de capital. El derecho a la vivienda ha sido sustituido por el derecho a endeudarse para comprar una vivienda, convirtiendo los hogares y los barrios en "la última frontera del capitalismo financiero de alto riesgo" (Rolnik, 2018). En este mismo sentido, García-Lamarca y Kaika (2016) sostienen que la vinculación del acceso a la vivienda con el acceso al crédito ha creado una conexión, cada vez más estrecha, entre las prácticas de vida cotidiana y las prácticas especulativas de los mercados inmobiliarios y financieros globales. En su trabajo muestran cómo, tras el estallido de la crisis en 2007, los cambios macroeconómicos y las políticas de financiarización de los mercados de vivienda han impactado en múltiples aspectos de la vida de la gente: reduciendo su capacidad para acceder a una vivienda o para tomar decisiones, rompiendo sus redes sociales, o repercutiendo sobre su salud. Se trata en definitiva de un proceso global de acumulación por desposesión (Harvey, 2004) cuyas repercusiones varían en función de los diferentes contextos (García-Lamarca y Kaika, 2016; Gutiérrez y Vives-Miró, 2018; Janoschka, 2016; Rolnik, 2018).

En Europa, la crisis financiera de 2007 se ha vinculado explícitamente con una crisis democrática (Kaldor y Selchow, 2013), cuya responsabilidad política se atribuye a la comunidad internacional y a las organizaciones supra-nacionales (BCE, FMI, etc.), pero también a los gobiernos nacionales, incapaces de proteger a los ciudadanos contra sus efectos (Armingeon y Guthmann, 2014; Della Porta, 2015; Flesher Fominaya, 2017; Langman, 2013).

Ante la ineficacia de la democracia representativa europea, ha emergido una oleada de movilizaciones que demanda una regeneración democrática profunda (Báez Urbina, 2013; Castells, 2012; Dekker y Feenstra, 2015; Flesher Fominaya, 2017). Este ciclo contrasta con el ciclo de movilizaciones precedente, que hacía una crítica abstracta contra el capitalismo global; en este nuevo ciclo de movilizaciones anti-austeridad o pro-democracia, la responsabilidad se traslada a las oligarquías políticas y financieras nacionales "que abusaron de su poder para el interés propio y en contra del interés directo de la gente, transfiriendo el costo de su "crisis / estafa' a las personas trabajadoras" (Flesher Fominaya, 2017, pp. 14-15); una crisis cuyos efectos (incremento del paro, recortes de los servicios públicos, desalojos, etc.) se evidencian y perciben 
en el entorno inmediato (familiares, vecinos, etc.), que afecta al ciudadano común y favorece la identificación de la gente como sujeto político. Los movimientos de toma de la calle son la expresión más emblemática de este proceso, popularizando eslóganes como: "No nos representan", "Somos el 99\%", "No somos anti-sistema, el sistema está contra nosotros". Se trata de una movilización global de movimientos locales que reclaman un proceso de democratización de las instituciones y que se hagan efectivos derechos constitucionales como el derecho a la vivienda (Álvarez, Zapata Campos y Zapata, 2015).

Las protestas de esta clase suelen traer como consecuencias un incremento de represión legal y/o la reconfiguración del paisaje político (Flesher Fominaya, 2017; Koopmans, 2004; Tarrow, 1998). En Europa se ha observado cómo, efectivamente, se han endurecido las legislaciones nacionales de países como España o Turquía, modificando sus marcos normativos para acallar la disidencia (Fernández-Savater y Flesher Fominaya, 2016). Asimismo, se han observado procesos de institucionalización de las movilizaciones sociales, ya sea mediante el surgimiento de nuevos partidos, como es el caso de Podemos en España, o por la adopción por parte de partidos existentes de las demandas sociales, como ha sido el caso de Syriza en Grecia (Martín, 2015; Lobera y Rogero-García, 2017).
Pese a la intensa relación entre movimientos sociales y partidos políticos desde hace más de doscientos años (Tilly, 2004), son escasos los estudios que la analizan (Kitschelt, 2006; Lobera y RogeroGarcía 2017; Martín, 2015; Van Cott, 2005), y más escasos aún los que abordan específicamente su capacidad para transformar las políticas de vivienda. Este trabajo tiene como objetivo utilizar el caso de España entre 2009 y 2019 para analizar la capacidad de estos procesos de lucha, que van desde la toma de la calle a la toma de las instituciones para transformar las políticas de vivienda existentes. En este sentido, se considera que el caso español puede ser paradigmático dada:

- La envergadura y el impacto de la crisis, con más de 700.000 ejecuciones hipotecarias ${ }^{3}$ desde su estallido en 2007.

- La vinculación entre crisis económica y las políticas de vivienda que se han venido implementando desde hace 80 años (Alguacil et ál., 2013; García-Lamarca y Kaika, 2016; Gutiérrez y Vives-Miró, 2018; Jiménez y Fernández, 2014; Naredo, 2010) y el entrelazamiento entre crisis financiera y deslegitimación política, particularmente tras la implementación de las políticas de austeridad.

- La magnitud del proceso de toma de la calle, y específicamente de la movilización social

3 Según datos del Consejo General del Poder Judicial del año 2018. 
por hacer efectivo el derecho a la vivienda que emergió en 2009 a través de la Plataforma de Afectados por las Hipotecas (PAH) (Álvarez et ál., 2015; Di Feliciantonio, 2017; Jiménez y Fernández, 2014).

- Así como el proceso posterior de toma de las instituciones a través del surgimiento de Podemos a finales de 2014, que cierra en 2019 su primer ciclo de representación política.

Por lo tanto, la toma de la calle se analiza a través de la PAH, y la toma de las instituciones a través de la evolución de Podemos, examinando su capacidad para transformar las políticas de vivienda existentes. Para dicho análisis se ha partido de la teoría de la "estructuración de la sociedad" de Giddens (1984), de particular interés para el estudio de fenómenos urbanos desde una perspectiva holística (Smith y García-Ferrari, 2012; Álvarez, Cabrera y Smith, 2019). Esta teoría parte de que estructura y agencia son mutuamente constituyentes, y que, por lo tanto la agencia, si bien se ve condicionada por su estructura, tiene capacidad para transformarla. Según Giddens, existen tres tipos de relaciones a través de las cuales se constituyen y transforman las prácticas sociales: (1) estructuras de autoridad, relacionadas con las formas de organización y las normas que las regulan; (2) estructuras de asignación, relacionadas con el flujo de recursos; y (3) sistemas de significado, relacionados con ideologías, racionalidades, discursos e imaginarios; tres tipos de relación, que interactúan entre sí.

En este estudio, analizamos las estructuras de autoridad que surgen al interior de estos movimientos que luchan por hacer efectivo el derecho a la vivienda, como alternativas, y cómo se articulan estas con las estructuras vigentes de toma de decisiones en torno a la vivienda. Esto conlleva propuestas de reestructuración de las relaciones entre la sociedad civil y el Estado hacia una democracia más participativa. El análisis de las estructuras de asignación se realiza a través de una interpretación de las propuestas de dichos movimientos con respecto a los marcos normativos y prácticas según los cuales se regula el acceso a la vivienda, y la relación económica entre el usuario y la vivienda. Se incluye una breve reflexión sobre cómo se gestionan recursos al interior de los movimientos aquí estudiados. Con respecto a los sistemas de significado se analizan los cambios en el imaginario que promueven estos movimientos, cambios que son fundamento para las reformas en las estructuras de autoridad y de asignación que proponen.

Esto nos permite plantear las siguientes cuestiones:

- ¿Qué modelos alternativos de estructura organizacional han propuesto la PAH y Podemos, y qué incidencia han tenido en las estructuras de gobierno que tienen competencias en vivienda? 
- ¿Cuáles son las propuestas de la PAH y Podemos con respecto al acceso a la vivienda, y hasta qué punto han influido en las políticas públicas de vivienda?

- ¿Qué cambios han logrado estos movimientos en la conceptualización de la relación entre ciudadano y vivienda, es decir, en el imaginario colectivo, tanto en la sociedad civil como en el Estado?

Respondiendo a estas preguntas, el artículo pretende atender también a la pregunta marco sobre la capacidad de estos procesos de lucha, que van desde la toma de la calle a la toma de las instituciones, para transformar las políticas de vivienda existentes.

El artículo se organiza como sigue. Tras esta primera sección de introducción, se presenta el método de investigación utilizado para la recogida de datos y análisis de los procesos de la PAH y Podemos, seguido de un somero análisis del contexto de la lucha por la vivienda en España (2009-2019), se continua con dos apartados donde se presentan los resultados obtenidos en cada caso de estudio respectivamente, $y$, por último, se extraen conclusiones a partir de una discusión cruzada de los resultados.

\section{Método}

En este artículo se ha partido del método de casos de estudio (Yin, 1994) con PAH y Podemos, a los cuales se les ha aplicado el marco analítico de la teoría de la estructuración de Giddens (1984) expuesto anteriormente. La información ha sido recogida y analizada, en las siguientes etapas (Frediani, 2007): inmersión previa, recogida sistemática y proceso de la información y, finalmente, extracción de conclusiones. La inmersión ha sido de utilidad para entender ambos procesos desde dentro, así como para establecer las conexiones entre los mismos. Entre 2011 y 2019, una de las investigadoras ha participado en una decena de asambleas de la PAH y ha realizado una veintena de entrevistas semi-estructuradas a activistas y profesionales que les apoyan (abogados). Paralelamente, entre 2014 y 2015, ha participado en el proceso de constitución de la nueva formación política, así como en reuniones quincenales de círculos sectoriales para la elaboración del programa de vivienda. Todo lo cual se realizó con el objetivo de entender, desde dentro de dichas estructuras, el modo en el que estas se conforman, asignan los recursos y configuran y modifican los sistemas de significado. Además, desde 2011 se ha venido 
recogiendo, de forma sistemática, la información que dichos actores publican en sus redes, así como otras informaciones publicadas en artículos de investigación o de divulgación. Finalmente, La información recogida y analizada a partir de la teoría de la estructuración de Giddens (1984) ha permitido extraer conclusiones respecto a la capacidad de ambos procesos para transformar las políticas de vivienda existentes.

\section{El contexto español: crisis financiera, deslegitimación política y movilización social}

El objetivo de este apartado es enmarcar el contexto político, financiero-inmobiliario y social en el que ha emergido y se ha desarrollado la lucha por el derecho a la vivienda (2009-2019).

\section{EMERGENCIA HABITACIONAL FRUTO DE LA IMPLEMENTACIÓN DE POLÍTICAS DE MERCANTILIZACIÓN Y FINANCIARIZACIÓN DE LA VIVIENDA}

En España se han producido más de 700.000 ejecuciones hipotecarias ${ }^{4}$ desde el estallido de la crisis

4 Según datos del Consejo General del Poder Judicial del año 2018. financiera en 2007, en contraste con los compromisos contraídos por el Estado Español en materia de derecho a la vivienda, tanto en su Constitución de 1978 como en el ámbito internacional ${ }^{5}$. Que España haya sido uno de los países más afectados por la crisis financiera responde a la implementación de un modelo inmobiliario-financiero-urbanístico, concebido no para satisfacer las necesidades de vivienda, sino como motor de la economía española. Un modelo que tiene sus raíces en el franquismo, que ha persistido en el régimen constitucional actual y que constituye una anomalía en la Unión Europea. Se trata de un proceso ampliamente estudiado (Alguacil et ál., 2013; Colau y Alemany, 2012; García-Lamarca y Kaika, 2016; Gutiérrez y Vives-Miró, 2018; López y Rodríguez, 2010; Naredo, 2010) que, dada su relevancia para esta investigación, pasamos a presentar brevemente a continuación:

- Durante la dictadura franquista (1939-1975) se asentaron las bases del modelo. Según el primer ministro de vivienda José Luis Arrese, se trataba de convertir un país de proletarios en un país de propietarios. Este mecanismo buscaba el control social, evitando la contestación del proletariado (Colau y Alemany, 2012; GarcíaLamarca y Kaika, 2016). En España en los años

5 El derecho a una vivienda adecuada está reconocido en el art. 47 de la Constitución Española. Además, está reconocido en el art.25 de la DUDH y en el art 11.1 del PIDESC, ratificados por el Estado español. 
50, en ciudades como Madrid o Barcelona, apenas un $12 \%$ de la población era propietaria de vivienda, mientras que en 2006 suponía el 85\% (Naredo, 2010). El sector de la construcción se convirtió, junto con el turismo, en el elemento clave del desarrollo económico, al tiempo que se desmantelaba el aparato productivo (López y Rodríguez, 2011).

- En las primeras décadas democráticas (19751999) se consolidó el modelo y se asentaron las bases para pasar de un país de propietarios a un país de hipotecados. El acceso a la vivienda en régimen de propiedad se estimuló mediante políticas de ayuda a la compra, desgravaciones fiscales, etc., lo que, a partir de 1981, se vio estimulado por la liberación del mercado hipotecario, así como por otras políticas de desincentivación del alquiler (véase el Decreto Boyer: Real Decreto-Ley 2, 1985). En los años 90, las hipotecas se convirtieron en el medio más atractivo para acceder a la vivienda, en particular para los hogares de bajos ingresos (López y Rodríguez, 2010). La relación entre deuda hipotecaria y PIB aumentó en 1998 a más del 250\% (García-Lamarca y Kaika, 2016).

- Entre 1999 y 2007 se produjo un crecimiento exponencial del sector de la construcción y se disparó la especulación inmobiliaria (Charnock, Purcell y Ribera-Fumaz, 2014). La adopción del euro (1999) y la consiguiente disminución de las tasas de interés (del 16\% en los 90 al 3\% en 2004) atrajeron la inversión extranjera directa en bienes inmuebles (entre 1986 y 2006 aumentó un 102\%). Entre 1997 y 2007, se construyeron más de 6 millones de viviendas, se concedieron hipotecas de hasta el 120\% del valor inmobiliario, y se incrementaron los precios hasta un 200\% (García-Lamarca y Kaika, 2016). El sector inmobiliario-financiero y las administraciones públicas transmitían el mensaje de que la vivienda era una inversión segura y que alquilar era malgastar el dinero (García-Lamarca y Kaika, 2016). El sector inmobiliario llegó a suponer el 70\% del crédito, el 30\% de la actividad económica y el 13\% del empleo (Colau y Alemany, 2012). Entretanto, la vivienda en régimen de alquiler se redujo al $13,5 \%$, la vivienda social prácticamente desapareció, suponiendo apenas un 2\% (Alguacil et ál., 2013), mientras la vivienda vacía alcanzaba los 3,4 millones, es decir, más de un 13\% del parque inmobiliario.

A finales de 2007, la vivienda se había convertido en el "activo más grande en la vida cotidiana de las personas". El 82\% de la población se consideraba "propietario de vivienda" cuando en realidad eran "propietarios de hipoteca" sobre-endeudados (entre 1997-2007 el endeudamiento paso de suponer el 55\% de la renta familiar disponible al 130\%), 
altamente dependientes de un sistema financiero global (en 2007 se estimaba que la deuda había sido bursatilizada en un 36\%) opaco, complejo e impredecible, e inmersos en un mercado laboral precarizado y dependiente del sector de la construcción (García-Lamarca y Kaika, 2016).

\section{CRISIS FINANCIERA, DESLEGITIMACIÓN POLÍTICA Y MOVILIZACIÓN SOCIAL}

Al estallar la crisis financiera de 2007, la situación empeoró al contraerse los mercados. En la segunda mitad de 2008, la economía española entró en recesión y se elevó la destrucción de empleo (la tasa de desempleo pasó de un 8\% en 2008 a un $26,6 \%$ en 2013), haciéndose imposible el pago de las hipotecas para cientos de miles de hogares (ODESC, 2013).

En 2011 se había desahuciado en torno a 300.000 familias, elevándose la tasa de pobreza al 20\% (Álvarez et ál., 2015). En este contexto, el gobierno del Partido Socialista Obrero Español (PSOE), con el apoyo del Partido Conservador (Partido Popular, PP), aprobó la modificación del artículo 135 de la Constitución Española, con el fin de anteponer el pago de la deuda al bienestar social ("El artículo 135 de la Constitución", 2014). Desde 2010, se estima que se recortaron más de 121 millones en salud, educación, pensiones y otros derechos sociales
(Fraguas, 2013), mientras las ayudas públicas a las entidades financieras españolas ascendieron a los 1.427.355 millones de euros (Cappa, 2013). En 2012, España se convirtió en uno de los países más rescatados de Europa (con ayudas públicas de 3,8 billones de euros) y, tras décadas de promoción y desregulación del mercado hipotecario, la central europea exigió solvencia a las entidades financieras rescatadas, lo que se llevó a cabo vendiendo estos activos a inversores extranjeros. En 2014, por ejemplo, 112.00 viviendas fueron vendidas a la firma Blackstone, a la mitad de su valor nominal (Munoz, 2014). Las personas se convirtieron en objetos financieros susceptibles de usar y tirar (Dean, 2012) y sus vidas cotidianas y su trabajo futuro pasaron a ser objeto de prácticas de especulación financiera. Se produjo un trasvase de la deuda, del sector privado al sector público, "socializando las pérdidas y asegurando la satisfacción de las cantidades prestadas a los bancos europeos" (Gutiérrez y Vives-Miró, 2018, p. 9). Como consecuencia de todo ello, entre 2007 y 2019 se produjeron más de 700.000 ejecuciones hipotecarias, y la deuda pública se triplicó ${ }^{6}$. Como afirman Colau y Alemany (2012), más que una crisis nos encontramos ante lo que parece una estafa reglada y amparada por la ley con consecuencias devastadoras para la mayoría de la población.

6 Según datos del INE. 
A esta situación de crisis se sumó un goteo continuo de casos de corrupción, que afectaban en mayor o menor medida a todos los partidos políticos. El consenso socio-político del llamado régimen del 78 se resquebrajó (Bescansa, 2015). La indignación de la sociedad española no hizo sino aumentar, al constatar las consecuencias de la crisis en su entorno próximo (familiares, vecinos), mientras presenciaban la impunidad de la mayoría de los corruptos y la falta de voluntad política para anteponer los derechos de las personas a los intereses financieros. En este contexto emergió la idea de que era necesario recuperar la soberanía popular. En mayo de 2011 bajo el eslogan ¡Democracia real Ya! Toma la calle se convocó una manifestación que no estaba apoyada por ningún sindicato o partido político, y que fue ignorada por los medios de comunicación, pero en Madrid y otras 50 ciudades españolas, decenas de miles de personas salieron a manifestarse. Apenas 5 meses después, se produjeron manifestaciones en más de 950 ciudades de 82 países del mundo (Castells, 2012).
"Ocurrió cuando nadie lo esperaba. En un mundo presa de la crisis económica, el cinismo político, (...) los magos de las finanzas pasaron de ser objeto de envidia pública a objeto de desprecio universal. Los políticos quedaron en evidencia como corruptos y mentirosos (...) la confianza (que es lo que cohesiona a una sociedad) se desvaneció (...) en los márgenes de un mundo que había llegado al límite de su capacidad para que los seres humanos convivieran y compartieran la vida con la naturaleza, los individuos volvieron a unirse para encontrar nuevas formas de ser nosotros." (Castells, 2012, p. 19)

La toma de las plazas, promovida en España por el denominado movimiento $15 \mathrm{M}$, se convirtió en un momento de catarsis colectiva: "la gente pasó de hablar de fútbol a hablar de lo realmente importante en sus vidas: la educación, la sanidad, la vivienda, el modelo económico, el modelo político, etc." (Activista de la PAH). La gente común se constituyó en sujeto político.

En este sustrato de crisis financiera-inmobiliaria, deslegitimación política y movilización social, emerge y se desarrolla el proceso de lucha por la 
vivienda en España (2009-2019) desde la toma de la calle, a través de la PAH, a la toma de las instituciones, a través de Podemos. A continuación, se presenta el análisis de los casos con el enfoque de Giddens (1984).

\section{La lucha por la vivienda en España (2009-2019) por medio de la toma de la calle: el caso de la PAH}

En 2006, el relator especial de la ONU sobre el Derecho a una Vivienda informó de que el derecho a la vivienda en España estaba siendo vulnerado (Kothari, 2008). Sin embargo, en 2009, las administraciones públicas españolas seguían negando que las familias tuvieran dificultades para hacer frente a las hipotecas. En una situación de vulneración del derecho a la vivienda y de abandono institucional, la sociedad civil española se organiza, y en 2009 se constituye la Plataforma de Afectados por las Hipotecas (PAH) con el objetivo de transformar un modelo con consecuencias devastadoras en todos los ámbitos: social, político, económico y medioambiental (Colau y Alemany, 2012). Pero en el corto plazo tiene que ofrecer una respuesta inmediata y concreta a una población abandonada por la administración y amenazada por las entidades financieras, que se siente angustiada, culpable y abatida.
A continuación, se presenta un análisis de la estructura organizativa, la asignación de recursos y el sistema de significado con el que la PAH encara sus objetivos.

\section{ESTRUCTURA ORGANIZATIVA EN EL CASO DE LA PAH}

La PAH se crea en Barcelona con el fin de ser reproducible, tanto en su estructura y en su asignación de recursos como sus sistemas de significado (Colau y Alemany, 2012). La irrupción de la PAH llegó con el $15 \mathrm{M}$ y con las asambleas locales que se crearon en todo el país. En 2012, la PAH contaba con 160 nodos locales y autónomos pero conectados entre sí a través de las redes sociales y la asamblea estatal de la $\mathrm{PAH}$, lo que les permite, adaptándolas al contexto local, apropiarse de las mismas prácticas y reivindicaciones. (Álvarez et ál., 2015). Apenas un año después, la PAH no solamente está plenamente instalada en todo el país, llevando a cabo acciones de manera coordinada, sino que ha logrado el apoyo del 80\% de la sociedad a sus demandas (Garea, 2013).

La PAH está impulsada por activistas de vivienda y cuenta con el apoyo altruista de profesionales (juristas, periodistas, etc.), asociaciones de vecinos, etc. Pero las afectadas por los procesos de ejecución son el eje central del movimiento. 
Las asambleas de personas afectadas y quienes les apoyan se reúnen semanalmente en un lugar fijo; empiezan siendo pequeñas, pero al crecer el problema y difundirse su existencia pasan a estar conformadas por cientos de personas (Álvarez et ál., 2015). Las asambleas se organizan de forma horizontal; en ellas, las afectadas comparten sus situaciones, se realizan formaciones, se comparte la información procedente de otras asambleas, se preparan acciones y se posibilita el encuentro gratuito con abogados, psicólogos, etc.

Las asambleas se convierten en el instrumento clave para unirse y perder el miedo (Castells, 2012). Se conforman como un espacio en el que apoyarse mutuamente e ir ganando confianza, un lugar en el que poder compartir y ser escuchado, en el que desculpabilizarse y empoderarse. Un espacio en el que las afectadas pasan de víctimas a sujetos políticos de transformación colectiva de un problema estructural (Colau y Alemany, 2012).

"Has pasado sola por un rodillo institucional (...) Te sientes fracasada y culpable (...) la primera vez que cuentas tú caso en la asamblea es como si te quitaras una mochila (...) en un mes, el cariño que te transmite la gente, hacer cosas en común (...) es transformador, sientes un hilo de esperanza que te va empoderando (...) Vas sacando toda tu rabia y te conviertes en más activista que los activistas (...) La asamblea te da fuerzas para tirar adelante, para hacer cosas que ni se te habría ocurrido que podías hacer." (Afectada de la Asamblea de Vivienda de Móstoles).

\section{ASIGNACIÓN DE RECURSOS EN EL CASO DE LA PAH}

La PAH se financia gracias a las donaciones de las personas y entidades que les apoyan; no recibe ni cuotas de socios, ni subvenciones, ni ningún otro tipo de financiación, lo que le da total autonomía con respecto del resto de instituciones.

Para lograr sus objetivos, la PAH realiza campañas y acciones relacionadas con la asignación de recursos en torno a la vivienda que contribuyen a hacer visibles las causas del problema, al tiempo que responden a las urgentes demandas de los afectados.

En un contexto de abandono institucional y vulneración sistemática del derecho a la vivienda, se pone en marcha la campaña Stop Desahucios con la que se ha logrado paralizar más de 2.000 desahucios. Además, gracias a la movilización social y a las presiones a las entidades financieras, se ha logrado negociar miles de alquileres sociales y de daciones en pago (es decir saldar la deuda entregando la vivienda, algo que no permite la ley hipotecaria vigente).

Y dado que el marco normativo actual deja sin amparo a los afectados, se inicia un largo proceso para forzar al parlamento español a aprobar una nueva Ley de vivienda estatal ("Ley de vivienda de la PAH", s.f.), que permita la paralización de los desahucios y la dación en pago, promueva los alquileres sociales, etc. Tras una recogida de firmas de más de un millón y medio, en febrero de 2013, después 
de dos años de lucha se consigue presentar en el Congreso una Iniciativa Legislativa Popular (ILP), no obstante, el Gobierno siguió sin reaccionar ${ }^{7}$. Por otra parte, se presiona a las administraciones públicas autonómicas y se logra que se aprueben leyes locales en Navarra (24/2013), Andalucía (4/2013), Canarias (2/2014) y Cataluña (24/2015). En términos generales, estos nuevos marcos reguladores reconocen la situación de emergencia social, plantean medidas para sancionar la vivienda vacía en manos de entidades financieras e inmobiliarias y proponen medidas para dar respuesta a las familias en riesgo de exclusión, como la paralización de los desahucios o la puesta en marcha de nuevos planes de vivienda autonómicos. En 2018, la PAH presenta nuevamente una ley de vivienda estatal con el fin de "acabar con la emergencia habitacional y sentar las bases de un nuevo modelo que garantice el derecho a la vivienda" (Martínez, 2018). Pero, una vez más, es vetada por la mayoría parlamentaria y no es ni siquiera admitida a debate.

Finalmente, ante la falta de un parque público de vivienda social, más de tres millones de viviendas vacías y miles de familias desahuciadas, se pone en marcha la Obra social la PAH (Figura 1). Esta campaña persigue un triple objetivo: (i) recuperar la función social de las viviendas vacías, y más concretamente las de aquellas que se encuentran en manos de entidades financieras procedentes de ejecuciones hipotecarias, (ii) garantizar que las familias no se quedan en la calle al tiempo que se agudiza la presión sobre las entidades financieras para que acepten la dación en pago, (iii) y, por último, forzar a las administraciones públicas a que adopten las medidas necesarias para garantizar el derecho a la vivienda recogido en la CE y ratificado en otros acuerdos internacionales.

En la actualidad, más de 2.500 personas han sido realojadas gracias a la Obra Social la PAH.

"Si el Estado nos ha abandonado (...) vamos a tomar y hacer, en lugar de pedir y esperar (...) primero te paro un desahucio y visibilizamos el conflicto social que realmente es (...). Y si nos echas te ocupamos un bloque (...). Cuando te lo quitan todo, cuando estás pensando en suicidarte (...) solo te queda pelear." (Activista de la Asamblea de Vivienda de Móstoles).

7 La ley hipotecaria española fue declarada ilegal por el Tribunal de Justicia Europeo. Si bien la reforma que se vio obligado a aprobar el Gobierno (ley 1/2013), sigue siendo insuficiente al mantener en la práctica la "deuda perpetua" (Observatorio ODESC, 2013), los desalojos forzosos y plantear la retroactividad en condiciones inviables en la práctica (véase: "Respuesta de la PAH”, (s.f.)) 


\section{FIGURA 1. EDIFICIO RECUPERADO "LA DIGNIDAD".}

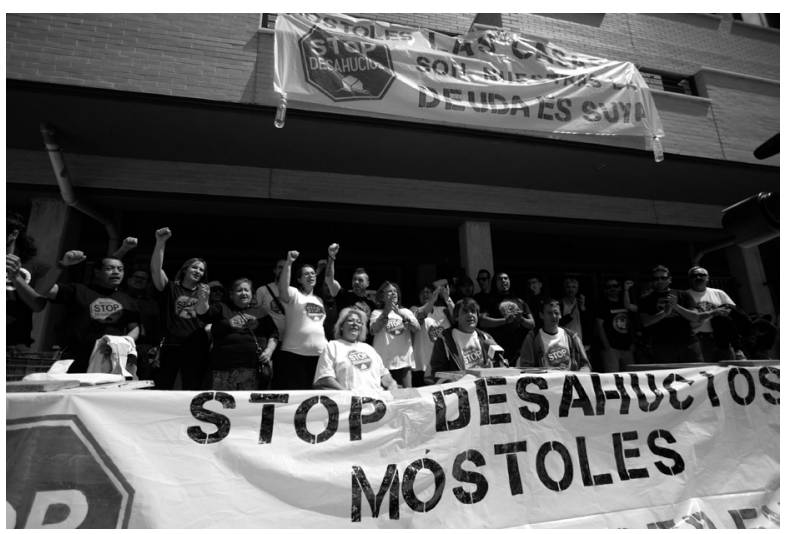

Fotografía de Alberto Astudillo, en Móstoles (25 de mayo 2014).

\section{Sistemas de significado en el caso de la PAH}

La PAH ha mostrado que "si nos organizábamos y manteníamos el rumbo, podíamos cambiar las cosas" (Colau y Alemany, 2013:42). Se ha logrado articular una respuesta colectiva a escala nacional. Los afectados han pasado de víctimas a sujetos políticos con capacidad para transformar la realidad: miles de desahucios paralizados, de personas realojadas, propuestas de ley de vivienda presentadas a las asambleas autonómicas y estatales, etc. La PAH ha logrado el apoyo del 80\% de la sociedad a sus demandas (Garea, 2013), ha transformado el imaginario colectivo (Castells, 2012) y ha desafiado el discurso del sistema capitalista, de que los derechos de la propiedad deben anteponerse al bien común y más concretamente al derecho a la vivienda (Harvey, 2012).

"Nombrar las realidades genera otra realidad. La gente graba lo que pasa con su móvil y lo difunde en las redes, y se ve cómo se saca a la gente de sus casas, a un niño o a un anciano (...). En este país no queremos 'ni casas vacías, ni gente sin casa." (Activistas de la PAH)

Las acciones de la PAH, y en particular la lucha porque se apruebe la Ley de vivienda estatal, muestran que hay alternativas y visibiliza que "¡Sí se puede! ¡Pero no quieren!” (Figura 2). Surge entonces una cuestión crucial: si se cuenta con propuestas viables y con el apoyo social de la mayoría, ¿cómo hacer para lograr que las instituciones las hagan efectivas? ¿Habrá que tomar las instituciones para hacerlas funcionar al servicio del bien común?

En enero de 2014, un año después de que a pesar de que se aceptara a trámite la ILP, nada cambiara en lo que al posicionamiento institucional se refiere, surge la nueva formación política Podemos, que, como veremos a continuación, incorporará en su programa político las demandas de vivienda de la PAH, elementos claves de su discurso e incluso a título individual algunos de sus representantes. "Dijeron en las plazas que sí se puede y nosotros decimos hoy que Podemos (...) disputarles las calles, donde están incómodos, y disputarles también sus lugares de poder, como las instituciones" (Declaraciones de los líderes de Podemos en Giménez 
San Miguel, 2014). En el siguiente apartado, se presentan los resultados del análisis del proceso de toma de las instituciones, su estructura, asignación de recursos, y sistemas de significado.

\section{La lucha por la vivienda en España (2014-2019) por medio de la toma de las instituciones: el caso de Podemos}

Podemos se constituye en enero de 2014, cuando se ha verificado que desde la movilización social no se logra que las instituciones recojan propuestas como la ley de vivienda estatal presentada por la $\mathrm{PAH}$, pese al fuerte respaldo social.

Podemos se presenta en la escena política con el objetivo de: "convertir la indignación en cambio político" (Sánchez y Gil, 2014), y se ofrece como una herramienta de auto-organización de la gente para la defensa de los derechos: techo, trabajo y pan, una reivindicación social ${ }^{8}$ que, como afirma Teresa Rodríguez, "pone en cuestión lo que son los privilegios de la clase dominante, el sistema financiero, las reformas laborales, la ley hipotecaria" (Rivero, 2014).

8 El lema de las marchas por la dignidad es: Techo, trabajo y pan.

\section{FIGURA 2. ¡SÍ SE PUEDE! ¡PERO NO QUIEREN!}

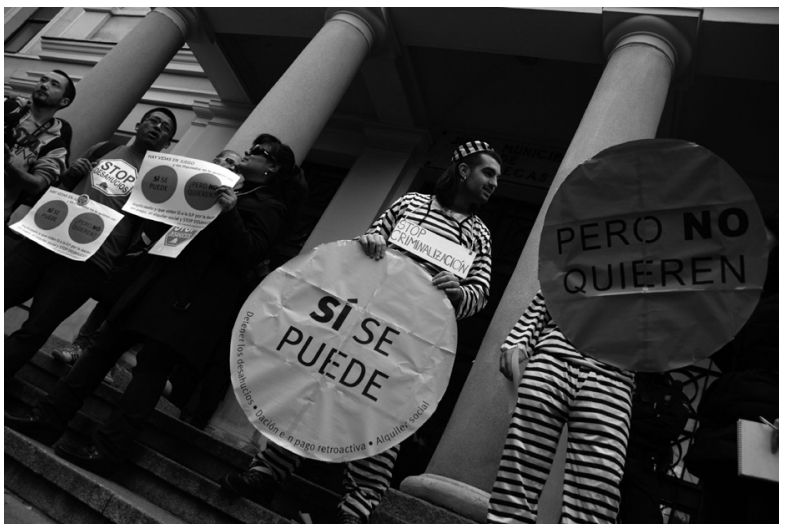

Fotografía de Alberto Astudillo, en Vallecas (Madrid), 3 de abril de 2013.

\section{ESTRUCTURA ORGANIZATIVA EN EL CASO DE PODEMOS}

La nueva formación arranca sin recursos económicos, pero con un importante capital mediático9 y social, como prueban las 50.000 firmas recogidas, para avalar su participación en las elecciones al Parlamento Europeo de 2014, en apenas 24 horas. En mayo de 2014, apenas cuatro meses tras su creación, Podemos irrumpe en la escena política con 1,2 millones de votantes (7,98\%), logrando

9 En diciembre de 2014 programas presentados por Pablo Iglesias, futuro Secretario General de PODEMOS, cuentan con más de 9 millones de seguidores. (Bollero, 2014). 
una representación de cinco eurodiputados en el Parlamento Europeo ("Elecciones europeas", 2014) y situándose como la cuarta fuerza política del país. En julio de 2014, Podemos cuenta con más de 600.000 seguidores lo que la consolida como la primera fuerza política en las redes sociales (Bollero, 2014), unas 10 veces más que los partidos tradicionales como PSOE y PP. Además, cuenta con cientos de "círculos" que se reúnen en asambleas por todo el territorio español.

Tras la asamblea fundacional, en octubre de 2014, se sucedieron las elecciones para los consejos ciudadanos de Podemos a nivel estatal, autonómico y municipal. Una vez constituidos estos consejos, se convoca para el 31 de enero de 2015 la Marcha del Cambio. Una movilización a la que se estima acudieron en torno a 100.000 personas, en la que se hace un llamamiento a la toma de las instituciones, a través de los comicios autonómicos y estatales: "vamos a ser los ciudadanos los que recuperemos las instituciones para ponerlas al servicio de nuestros intereses, y no de los intereses de la minoría privilegiada que nos gobierna" (Podemos, 2015). En apenas dos años tras su creación, Podemos se instala con 71 escaños en el congreso de los diputados, en confluencia con Izquierda Unida IU (junio 2016) y se convierte en la tercera fuerza política del país.
En lo que a su estructura organizativa se refiere, Martín (2015) sostiene que desde el inicio Podemos imita o incorpora aspectos organizativos de los movimientos sociales y crea un vínculo no institucionalizado. Algo que se refleja, por ejemplo, en el primer consejo ciudadano de Podemos, constituido en un $37 \%$ por personas provenientes de movimientos sociales. Por otra parte, se produce una cierta superposición entre los círculos de Podemos (unidades básicas de participación social) y las asambleas locales del 15M.

En lo que a los procesos de toma de decisiones se refiere, si bien se promueve la participación en la elaboración de listas y programas electorales, se apuesta desde el inicio por un modelo que defiende "el pragmatismo y un cierto verticalismo" (defendido por el Secretario General y sus seguidores). Esta opción supondrá un conflicto al interior del partido con otro sector que defiende la pluralidad y la horizontalidad (apoyada por la líder del partido en Andalucía y los "anti-capitalistas"). Estas tensiones se evidenciaron públicamente durante la Asamblea constituyente (octubre de 2014 en Vistalegre) y se han visto reflejadas en todos los órganos del partido, desde los ejecutivos a los círculos locales (Martín, 2015). 


\section{ASIGNACIÓN DE RECURSOS EN EL CASO DE PODEMOS}

Podemos parte desde el inicio de la voluntad de no depender de las entidades económicas y financieras, por lo que se financia desde el principio de sus colaboradores y donantes, dentro de los límites legales estableciendo para dichas contribuciones. Además, desde su compromiso por la transparencia, hace públicos todos sus ingresos y gastos ${ }^{10}$. Con respecto a la asignación de recursos en torno a la vivienda, desde el inicio Podemos recoge las reivindicaciones provenientes de la movilización social y, apenas finaliza la primera asamblea constituyente (octubre, 2014), se aprueban cinco resoluciones ${ }^{11}$ encaminadas a: luchar contra la corrupción, auditar la deuda y defender los derechos básicos como vivienda, salud y educación.

En lo que se refiere específicamente a las políticas de vivienda, en sus programas electorales tanto de 2015 como de 2019, se recogen las principales demandas de la PAH, es decir: la prohibición de los desalojos forzosos sin alternativa habitacional; la dación en pago con carácter retroactivo; el acceso garantizado a los suministros básicos; reformar la ley de arrendamientos urbanos, para facilitar un alquiler estable y asequible; así como un conjunto de medidas para regular el mercado inmobiliario y la promoción de la vivienda social (artículos 129 -135, del programa de 2015 y 187-196, del programa de 2019).

No obstante, hasta el momento Podemos no ha logrado la fuerza política suficiente para entrar en el Gobierno, ni por lo tanto ha podido implementar su programa político; desde la oposición ha defendido la ley estatal de vivienda presentada por la $\mathrm{PAH}$, y que los dictámenes de Naciones Unidas en relación a la vivienda fueran vinculantes ${ }^{12}$. Según declaraciones de un activista de la PAH, "en el último año, que ha tenido más capacidad de influir"13, han forzado al PSOE a modificar la ley de arrendamientos urbanos".
10 Fuente: http://transparencia.podemos.info/

11 Fuente: http://asambleaciudadana.podemos.info/resoluciones.
12 Como el dictamen del 20/06/2017 (véase “Javier Rubio explica el dictamen", 2017).

13 En 2018, el PSOE forma gobierno, tras presentar una moción de censura al PP, al conocerse la sentencia de la Audiencia Nacional que afirmaba que el PP había ayudado a establecer "un sistema genuino y efectivo de corrupción institucional". El apoyo de Podemos fue decisivo para que prosperará la moción (Cruz, 2018). 


\section{Sistemas de significado en el caso de Podemos}

Podemos reivindica la construcción de una democracia real y ha instalado en el imaginario colectivo la idea de que "si la gente no hace política te la hacen otros; y si te la hacen otros te roban los derechos, la democracia y la cartera" (según declaraciones públicas de Pablo Iglesias, en mayo de 2014). Según Martín (2015), el principal éxito de Podemos reside precisamente en la apropiación del discurso creado por los movimientos sociales, que habían logrado un amplio apoyo social y que permiten a Podemos aglutinar la fuerza de la indignación en torno a un eje político ni de izquierdas ni de derechas. Esto se observa en la utilización del término casta para referirse y diferenciarse de los políticos que no nos representan (eslogan del 15M), o la elección de la democracia (Figura 3) en tanto que el eje estructurante del programa de 2015. Un ejemplo de esta apropiación, no sólo del discurso sino también de las reivindicaciones y la lucha llevada a cabo por los movimientos sociales, es la intervención final del debate televisivo de Pablo Iglesias en 2015 ("Elecciones generales", 2015), en el que por una parte recoge la indignación en relación a los casos de corrupción que afectan a todos los partidos, y por otra reivindica la lucha y la esperanza de la ola de movilizaciones: "sonrían al $15 \mathrm{M}$, a las plazas, a quienes paraban desahucios (...) sonrían, que ¡sí se puede!".
FIGURA 3. LA "GENTE" REIVINDICA DEMOCRACIA REAL.

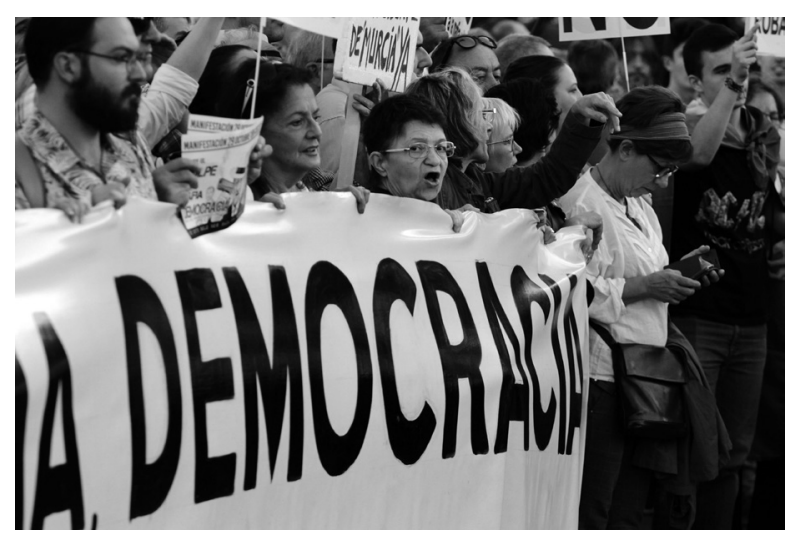

Fotografía de Alberto Astudillo, en Madrid, 29 de octubre 2016.

El éxito de Podemos, primero en las elecciones europeas y posteriormente en las generales, genera un "momento de euforia" (Lobera y Rogero-García, 2017). Activistas y electores vislumbran la posibilidad de que las demandas sociales puedan ser por fin acogidas en las instituciones.

Podemos logra quebrar el bipartidismo, si bien, como afirma Toussaint (2015), "tomar las instituciones" no equivale a "tomar el poder real" si el apoyo es insuficiente para implementar su programa político.

En las elecciones de 2019, Podemos afronta la división interna en relación a dos modelos organizativos 
en pugna, y su falta de capacidad real para acometer las reformas necesarias para atender a las urgencias sociales de techo, trabajo y pan.

Como advierte Kitschelt (2006), estos partidosmovimiento son de una gran inestabilidad por la relevancia de las cuestiones en torno a las cuales surgieron, así como por su capacidad para llevar a cabo sus propuestas y de transformar la realidad. En las elecciones de 2019, Podemos pierde el 40\% de sus escaños, que gana el PSOE; sin que se logré conformar un gobierno progresista en coalición.

\section{Conclusiones}

Desde el estallido de la crisis en 2007 se estima que se han producido más de 700.000 ejecuciones hipotecarias ${ }^{14}$ en España, un hecho que contrasta con los compromisos jurídicos contraídos por el Estado Español en materia de derecho a la vivienda. Se ha dado un proceso de acumulación por desposesión (Harvey, 2004; Janoschka, 2016) fruto de las políticas de vivienda que han venido implementándose en España desde hace más de 80 años (Alguacil et ál., 2013; Gutiérrez y Vives-Miró, 2018; Naredo, 2010).

En este contexto, se ha analizado el proceso de lucha por hacer efectivo el derecho a la vivienda en España entre 2009 y 2019, desde la toma de la calle, estudiándose la PAH a la toma de las instituciones, analizado a través de Podemos, así como la capacidad de ambos para transformar las políticas de vivienda existentes. Para dicho análisis se ha partido de la teoría de la estructuración de Giddens (1984). Esto ha permitido extraer las siguientes conclusiones, en relación a ambos procesos y a las preguntas planteadas al inicio:

- En lo que respecta a los modelos alternativos de estructura organizacional, la PAH adopta un modelo horizontal de nodos locales articulados entre sí. Y si bien Podemos imita ciertos aspectos organizativos de los movimientos sociales (Martín, 2015), defiende una postura centralizada y más verticalista en los procesos de toma de decisiones, lo que ha supuesto un conflicto al interior del partido. La irrupción de Podemos en el panorama político ha contribuido a reemplazar el bipartidismo con un sistema multipartido (tendencia también en otros países europeos), y la victoria de coaliciones en las que se integra Podemos en algunas municipalidades ha dado lugar a procesos de transformación organizativa a nivel local. En general, las actividades de la PAH y Podemos no han logrado alterar el modelo organizativo predominante en torno a la vivienda en España.

14 Según datos del Consejo General del Poder Judicial del año 2018. 
- En lo que a la asignación de los recursos se refiere, y atendiendo a la pregunta sobre las propuestas de la PAH y Podemos con respecto al acceso a la vivienda y las políticas públicas de vivienda, la PAH ha logrado articular una respuesta colectiva a escala nacional, paralizando desahucios, negociando alquileres sociales $y$ de daciones en pago, y realojando a afectados. Al tiempo que se ha presentado una ley estatal de vivienda, y se ha logrado la aprobación de leyes autonómicas como la ley catalana (24/2015). Podemos, por su parte, ha recogido las reivindicaciones de la Ley estatal de vivienda presentada por la PAH. Sin embargo, hasta el momento no ha logrado la fuerza política suficiente como para poder implementar su programa político.

- En lo que a los sistemas de significado se refiere, los mayores cambios logrados por estos movimientos han sido en la conceptualización de la relación entre ciudadano y vivienda, es decir, en el imaginario colectivo. La PAH ha mostrado mediante sus acciones que la realidad es transformable, que la gente común puede convertirse en sujeto político. Ha contribuido a visibilizar las causas estructurales de la crisis y sus consecuencias devastadoras para la mayoría de la población (Colau y Alemany, 2012), ha logrado el apoyo del $80 \%$ de la sociedad a sus demandas y ha desafiado el discurso del sistema capitalista de que los derechos de la propiedad deben anteponerse al bien común, y más concretamente al derecho a la vivienda (Harvey, 2012). Martín (2015) sostiene que el éxito de Podemos ha residido precisamente en la apropiación del discurso creado por los movimientos sociales, que habían logrado un amplio apoyo social. El auge de Podemos en 2105 genera un momento de euforia (Lobera y Rogero-García, 2017) que apunta a que se puedan, por fin, implementar las reivindicaciones sociales. Sin embargo, Podemos no alcanza la fuerza política suficiente para hacerlas efectivas y en 2019 pierde el 40\% del electorado. Según Kitschelt (2006), la inestabilidad de estos partidos-movimiento está vinculada a su capacidad para implementar sus programas políticos.

Con esto regresamos a la pregunta marco sobre la capacidad de estos procesos de lucha, que van desde la toma de la calle a la toma de las instituciones, para transformar las políticas de vivienda existentes. En el caso español, vemos que la toma de la calle llevó a la transformación del imaginario colectivo, a mostrar que hay alternativas y que una sociedad organizada y con objetivos claros puede cambiar las cosas (Colau y Alemany, 2013). La toma de las instituciones vislumbra la posibilidad de que las demandas sociales puedan ser por fin acogidas en las instituciones. La articulación entre la toma de la calle y la toma de las instituciones se ha 
producido a través del aprovechamiento por parte de Podemos de la movilización social desencadenada desde el 15-M, del discurso organizativo de los movimientos sociales (entre otros la PAH), y del programa de vivienda de la PAH, así como la cooptación de algunos de sus líderes. Sin embargo, a pesar de que Podemos ha llevado a la esfera institucional la lucha por transformar las políticas de vivienda existentes, no ha sido capaz de conseguir dicha transformación. Por un lado, las fuerzas estructurales existentes y el poder establecido han limitado drásticamente el alcance de estas transformaciones, quedando patente que ¡Sí se puede! ¡Pero no quieren!. Y por otro, al institucionalizarse, Podemos ha entrado en las dinámicas de rivalidad y posicionamiento que se producen entre los partidos políticos a la hora de formar gobierno o influir en los programas de gobierno, de tal manera que no ha conseguido la necesaria ampliación del apoyo hacia las propuestas de transformación de las políticas de vivienda entre otros partidos.

Si bien los resultados de esta investigación son dependientes de su contexto, el método empleado podría ayudar a entender otras situaciones similares de crisis financiera-inmobiliaria, deslegitimación política y movilización social. Por ejemplo, en Europa se pueden encontrar paralelos en países tales como Grecia o Irlanda, con sus particularidades. Además, en el contexto español, se considera que los resultados del análisis de las diferentes estructuras a escala nacional, podrían ser un punto de partida para análisis similares a escala municipal, con el fin de responder de forma territorializada a la pregunta marco planteada en esta investigación.

Este artículo pone de relieve que sigue vigente la necesidad de transformar un modelo que ha tenido consecuencias devastadoras en todos los ámbitos: social, político, económico y medioambiental (Colau y Alemany, 2012). Y el reto parece seguir siendo tomar el poder real (Toussaint, 2015), en un contexto de relaciones de poder desigual (Jenkins, Smith y Wang, 2007). O, dicho de otro modo, construir un nosotros (Flesher Fominaya, 2017) con capacidad para evitar que la vida cotidiana de millones de personas se siga considerando objeto de prácticas de especulación financiera susceptibles de usar y tirar (Dean, 2012).

\section{Referencias bibliográficas}

Alguacil, A, Alguacil, J., Arasanz, J., Fernández, G., Paniagua, J, Olea, S., y Renes, V. (2013). La vivienda en España en el siglo XXI. Diagnóstico del modelo residencial y propuestas para otra política de vivienda. Madrid: Cáritas; Fundación FOESSA.

Álvarez de Andrés, E., Cabrera, C., y Smith, H. (2019). Resistance as resilience: A comparative analysis of state-community conflicts around selfbuilt housing in Spain, Senegal and Argentina. Habitat International, 86, 116-125. doi:10.1016/j. habitatint.2019.03.003 
Álvarez de Andrés, E., Zapata Campos, M. J., y Zapata, P. (2015). Stop the evictions! The diffusion of networked social movements and the emergence of a hybrid space: The case of the Spanish mortgage victims group. Habitat International, 46, 252-259. doi:10.1016/j.habitatint.2014.10.002

Armingeon, K. y Guthmann, K. (2014). Democracy in crisis? The declining support for national democracy in European countries, 2007-2011. European Journal of Political Research, 53(3), 423-442. doi:10.1111/1475-6765.12046

Báez Urbina, F. A. (2013). Acción colectiva y movimientos de oposición ciudadana como contralores de decisiones gubernamentales: Una mirada desde la sociología analítica. Revista INVI, 28(79), 97122. doi:10.4067/S0718-83582013000300004

Bescansa, C. (2015). Aceleración del tiempo histórico [video]. Recuperado de https://www.youtube.com/ watch?v=_GZVkB_KfXA

Bollero, D. (2014, 16 de agosto). Podemos se consolida como la primera fuerza política en redes sociales. Público. Recuperado de https://www.publico.es/ politica/consolida-primera-fuerza-politica-redes. html

Cappa, M. (2013, 10 de octubre). La auditoría ciudadana cifra en 1,5 billones la ayuda a la banca. $\mathrm{La}$ Marea. Recuperado de https://www.lamarea. com/2013/10/10/la-ayuda-real-la-banca-espanola-ha-sido-de-15-billones-segun-la-facd/

Castells, M. (2012). Networks of outrage and hope. Social movements in the Internet age. Cambridge: Polity.
Charnock, C., Purcell, T., y Ribera-Fumaz, R. (2014). The limits to capital in Spain: Crisis and revolt in the European South. London: Palgrave Macmillan.

Colau, A. y Alemany, A. (2012). Vidas hipotecadas. Barcelona: Angle.

Colau, A. y Alemany A. (2013). ¡Sí se puede! Crónica de una pequeña gran victoria. Barcelona: Destino.

Cruz, M. (2018, 1 de junio). Pedro Sánchez, presidente del Gobierno tras triunfar la moción de censura contra Rajoy. El Mundo. Recuperado de https:// www.elmundo.es/espana/2018/06/01/5b1112342 68e3e9f618b4658.html

Dean, J. (2012). The communist horizon. London: Verso

Dekker, P. y Feenstra, R. A. (2015). Activism and civil society: Broadening participation and deepening democracy. Recerca: Revista de Pensament i Anàlisi, (17), 7-13. doi:10.6035/Recerca.2015.17.1

Della Porta, D. (2015). Social movements in times of austerity: Bringing capitalism back into protest analysis. London: John Wiley \& Sons.

Di Feliciantonio, C. (2017). Social movements and alternative housing models: Practicing the "politics of possibilities" in Spain. Housing, Theory and Society, 34(1), 38-56. doi:10.1080/14036096.2016.12204 21

El artículo 135 de la Constitución, antes y después de la reforma de 2011. (2014, 24 de noviembre). El País. Recuperado de: https://elpais.com/politica/2014/11/24/actualidad/1416849910_452980. html 
Elecciones europeas 2014: resultados. (2014, 25 de mayo). El Diario.

Elecciones generales. (2015, 8 de diciembre). Antena 3. Recuperado de http://www.antena3.com/especiales/noticias/elecciones-generales/2015/debate/ iglesias-quiero-pedirles-dos-cosas-que-olvidenque-sonrian_2015120800008.html

Fernández-Savater, A. y Flesher Fominaya, C. (Eds.). (2016). Roundtable: Life after the squares: Reflections on the consequences of the Occupy Movements. Social Movement Studies, 16(1), 119-151. do i:10.1080/14742837.2016.1244478

Flesher Fominaya, C. (2017). European anti-austerity and pro-democracy protests in the wake of the global financial crisis. Social Movement Studies, 16(1), 1-20. doi:10.1080/14742837.2016.1256193

Fraguas, T. (2013, 10 de diciembre). ¿Cuánto ha recortado ya el PP? La Marea. Recuperado de https://www.lamarea.com/2013/12/10/ cuanto-ha-recortado-ya-el-pp/

Frediani, A. A. (2007). Housing freedom, Amartya Sen and urban development policies -squatter settlement upgrading in Salvador da Bahia. (Ph.D. thesis, sin publicar). Oxford Brookes University, Oxford, Inglaterra.

García-Lamarca, M. y Kaika, M. (2016). "Mortgaged lives": the biopolitics of debt and housing financialisation. Transactions of the Institute of British Geographers, 41(3), 313-327. doi: 10.1111/tran.12126

Garea, F. (2013, 16 de marzo). Los españoles confían mucho más en los movimientos sociales que en los políticos. El País. Recuperado de https://elpais.com/politica/2013/03/16/actualidad/1363470095_882443.html

Giddens, A. (1984). The constitution of society: Outline of the theory of structuration. Cambridge, UK: Polity Press.

Giménez San Miguel, L. (2014, 17 de enero). Pablo Iglesias presenta Podemos como "un método participativo abierto a toda la ciudadanía". Público. Recuperado de https://www.publico.es/politica/pabloiglesias-presenta-metodo-participativo.html

Gutiérrez, A. y Vives-Miró, S. (2018). Acumulación de viviendas por parte de los bancos a través de los desahucios: geografía de la desposesión de vivienda en Cataluña. EURE, 44(132), 5-26. doi:10.4067/s0250-71612018000200005

Harvey, D. (2004). The "new" imperialism: Accumulation by dispossession. Socialist Register, 40: 63-87.

Harvey, D. (2012). Rebel cities: From the right to the city to the urban revolution. London: Verso.

Janoschka, M. (2016). Gentrificación, desplazamiento, desposesión: procesos urbanos claves en américa latina. Revista INVI, 31(88), 27-71. doi:10.4067/ S0718-83582016000300002

Javier Rubio explica el dictamen del PIDESC de $\mathrm{Na}$ ciones Unidas. (2017). PAH Madrid. Recuperado de https://pahmadrid.org/javier-rubio-explica-eldictamen-del-pidesc-de-naciones-unidas/

Jenkins, P., Smith, H., y Wang, Y. P. (2007). Planning and housing in the rapidly urbanising world. New York: Routledge. 
Jiménez, C. y Fernández, C. (2014). Casas sin gente, gente sin casas: el fracaso del modelo inmobiliario español. Revista INVI, 29(82), 133-155. doi:10.4067/S0718-83582014000300005

Kaldor, M. y Selchow, S. (2013). The 'bubbling up' of subterranean politics in Europe. Journal of Civil Society, 9(1), 78-99. doi:10.1080/17448689.2013 784501

Kitschelt, H. (2006). Movement parties. En Handbook of party politics. Londres: Sage

Koopmans, R. (2004). Protest in time and space: The evolution of waves of contention. En D. A. Snow, S. A. Soule, y H. Kriesi, (Eds.), The Blackwell Companion to Social Movements (pp. 19-46). London: John Wiley \& Sons.

Kothari, M. (2008). Promotion and protection of all human rights, civil, political, economic, social and cultural rights, including the right to development (A/ HRC/7/16/Add.2). United Nations.

Langman, L. (2013). Occupy: A new new social movement. Current Sociology, 61(4), 510-524. doi:10.1177/0011392113479749

Ley de vivienda de la PAH. (s.f.). Recuperado de https:// las5delapah.com/

Lobera, J., y Rogero-García, J. (2017). Medición de la cristalización electoral de un movimiento de protesta: De la indignación al voto. Empiria, (38), 151-176. doi:10.5944/empiria.38.2018.19715
López, I. y Rodríguez, E. (2010). Fin de ciclo. Financiarización, territorio y sociedad de propietarios en la onda larga del capitalismo hispano (1959-2010). Madrid: Traficantes de Sueños.

López, I. y Rodríguez, E. (2011). The Spanish model. New Left Review, (69), 5-28.

Martín, I. (2015). Tres modelos de partido-movimiento. RES Revista Española de Sociología, (24), 111-118.

Martínez, G. (2018). La PAH lleva su Ley de Vivienda al Congreso de los Diputados. El Salto. Recuperado de https://www.elsaltodiario.com/vivienda/ la-pah-lleva-su-ley-de-vivienda-al-congreso

Munoz, M. (2014, 17 de julio). Blackstone buys CatalunyaCaixa Mortgages for EU3.6 billion. Bloomberg. Recuperado de https://www.bloomberg.com/ news/articles/2014-07-16/blackstone-said-tobuy-catalunya-banc-s-distressed-mortgages

Naredo, J. M. (2010). El modelo inmobiliario español y sus consecuencias. Boletin CF+S, (44), 13-27.

Observatorio ODESC. (2013). Emergencia habitacional en el Estado español. La crisis de las ejecuciones hipotecarias y los desalojos desde una perspectiva de derechos humanos. Recuperado de http://observatoridesc.org/sites/default/files/2013-informe_habtitatge-17dic.pdf

Podemos. [iSí se puede!]. (2015, 31 de enero). La marcha del cambio [video]. Recuperado de https://www. youtube.com/watch?v=M4gdeJm67jw 
Real Decreto-Ley 2. Sobre medidas de política económica. Boletín Oficial del Estado (111). Madrid, España, 30 de abril de 1985.

Respuesta de la PAH a la Ley $1 / 2013$ del Partido Popular: una ley inútil, ilegal y que vulnera derechos humanos. (s.f.). Recuperado de https://afectadosporlahipoteca.com/2013/05/28/respuest-pah-ala-ley-12013-clausulas-abusiva-partido-popular-que-vulnera-derechos-humanos/

Rivero, J. (2014, 22 de mayo). Podemos surge la necesidad de algo diferente. Diagonal. Recuperado de https://www.diagonalperiodico.net/ panorama/22989-podemos-surge-la-necesidadpor-algo-diferente.html

Rolnik, R. (2018). La guerra de los lugares: La colonización de la tierra y la vivienda en la era de las finanzas. Barcelona: Descontrol.

Sánchez, J. L. y Gil, A. (2014, 14 de enero). Pablo Iglesias prepara su candidatura a las europeas: "Me han pedido que dé el paso". El Diario. Recuperado de https://www.eldiario.es/politica/Pablo-IglesiasJuan-Carlos-Monedero-candidato-europeas-podemos_0_216278861.html

Smith, H. y García Ferrari, M. S. (2012). Negotiating city-building in waterfront communities around the North sea: An analytical framework. En H. Smith y M. S. García Ferrari (Eds.), Waterfront regeneration: Experiences in city-building (pp. 17-32). Abingdon, UK: Routledge.
Tarrow, S. (1998). Power in movement: Social movements and contentious politics. Cambridge: Cambridge University Press.

Tilly, C. (2004). Contention and democracy in Europe, 1650-2000. Cambridge: Cambridge University Press.

Toussaint, E. (2015, 12 de febrero) Syriza y Podemos: ¿una vía hacia el poder del pueblo? Público. Recuperado de https://www.publico.es/opinion/articulos/syriza-y-via-hacia-del.html

Van Cott, D. L. (2005). From movements to parties in Latin America. The evolution of ethnic parties, Cambridge: Cambridge University Press.

Yin, R. K. (1994). Case study research: Design and methods. Thousand Oaks (CA): Sage. 